\title{
ASSESSMENT OF SENTINEL-5P PERFORMANCE FOR GROUND-LEVEL AIR QUALITY MONITORING: PREPARATORY EXPERIMENTS OVER THE COVID-19 LOCKDOWN PERIOD
}

\author{
D. Oxoli ${ }^{1}$, J. R. Cedeno Jimenez ${ }^{1 *}$, M. A. Brovelli ${ }^{1}$ \\ ${ }^{1}$ Department of Civil and Environmental Engineering, Politecnico di Milano, P.zza Leonardo da Vinci 32, 20133, Milan, Italy - \\ (daniele.oxoli, maria.brovelli)@ polimi.it, jesusrodrigo.cedeno@mail.polimi.it
}

\section{Commission IV}

KEY WORDS: Air Quality, Sentinel-5P, Earth Observations, Air Pollution Patterns, Nitrogen Dioxide

\begin{abstract}
:
Scientific evidence has demonstrated that deterioration of ambient air quality has increased the number of deaths worldwide by appointing air pollution among the most pressing sustainability concerns. In this context, the continuous monitoring of air quality and the modelling of complex air pollution patterns is critical to protect population and ecosystems health. Availability of air quality observations has terrifically improved in the last decades allowing - nowadays - for extensive spatial and temporal resolved analysis at both global and local scale. Satellite remote sensing is mostly accountable for this data availability and is promising to foster air quality monitoring in support of traditional ground sensors measurements. In view of the above, this study compares observations from the Sentinel-5P mission of the European Copernicus Programme (the most recent Earth Observation platform providing open measurements of atmospheric constituents) with traditional ground measurements to investigate their space and time correlations across the Lombardy region (Northern Italy). The correlation analysis focused on nitrogen dioxide. The use of data collected during the COVID-19 pandemic allowed for a parallel exploration of the lockdown effects on nitrogen dioxide emissions. Results show a marked decrease in nitrogen dioxide concentrations during the lockdown and an overall strong positive correlation between satellite and ground sensors observations. These experiments are preparatory for future activities that will focus on the development of satellite-based air quality local prediction models, aiming at improving the granularity of the ground-based information available for air quality monitoring and exposure modelling.
\end{abstract}

\section{INTRODUCTION}

Atmospheric pollution has been largely recognized by the scientific community as a primary threat to human health and ecosystems and represents a pressing sustainability concern, directly connected to many Sustainable Development Goals (e.g. 3.9 and 11.6) (Rafaj et al., 2018). Several studies in the literature have addressed the analysis of this complex phenomenon by paving the way to the development of global and local policies to mitigate its negative effects (Kuklinska et al., 2015). These studies have provided consolidated evidence linking the increase in the concentration of widely diffused pollutants, including coarse and fine particulate matter $\left(P M_{10}\right.$ and $\left.P M_{2.5}\right)$, ozone $\left(\mathrm{O}_{3}\right)$, nitrogen dioxide $\left(\mathrm{NO}_{2}\right)$, and sulfur dioxide $\left(\mathrm{SO}_{2}\right)$, with the growth in hospital admissions and cardiovascular diseases. As a result, it has been proved that air quality deterioration has increased the number of deaths worldwide even at low levels of exposure (Brunekreef, Holgate, 2002). Most of the air pollutants and greenhouse gases have increased their presence in the lower atmosphere during the last decades as a directed result of the growth of core production and consumption systems such as road transport, power plants, industry, farming, and households (Duque et al., 2016). In this context, the continuous improvement of air quality monitoring methods and techniques features a critical aspect to protect population and ecosystems health.

Air quality monitoring is traditionally performed utilizing fixed ground sensors networks, which represents the primary air quality data source to many national and local authorities. The use

\footnotetext{
${ }^{*}$ Corresponding author
}

of permanent ground sensors networks allows for continuous measures thus enabling computations of air quality indicators required by the current legislation (WHO, 2006). Nevertheless, the exclusive use of permanent ground sensors denotes several limitations to optimal monitoring of air pollutants (Kim et al., 2020). The main reason is that observations from fixed ground sensors limit the spatial granularity and coverage of the measurements. This often results in patchy monitoring across regions covered by the sensor networks, thus impacting on exposure assessments which on the contrary would require finescale and spatialized observations to be performed. Moreover, ground sensors networks imply high installation and maintenance costs and the increase sensors densities to achieve better spatial coverage is often unfeasible to many local networks managing authorities.

According to the above, modern air quality research has focused on two main aspects that can be summarized in the design of cost-effective technologies to improve air quality monitoring capacity and accuracy and, in parallel, to the enforcement of methodological component connected to air quality metrics, source apportionment analysis, and exposure models (Heal et al., 2012). In fact, atmospheric pollution monitoring has been empowered in recent years with satellite sensors that are capable of providing daily high-resolution air pollutants estimates on a global scale. The growing availability of satellite atmospheric observations that are distributed as open-data, above others the recent Sentinel-5P mission of the European Copernicus Programme (Koch, Bydekerke, 2018), enforces the interest in exploiting satellite remote sensing to support air quality monitoring. Despite applications on a global scale, the spatial and 
temporal resolution provided by the Sentinel-5P is promising for employment to air quality monitoring also at a local scale.

With this in mind, this work presents a preliminary investigation on the contribution of satellite estimates as an alternative or a complementary data source for local air quality monitoring. Observations from the Sentinel-5P were compared with authoritative fixed ground sensors observations from the Lombardy region (Northern Italy) as a case study. The main objective was to quantify space-time correlations and trade-offs between satellite estimates and ground measurements. In the first place, the correlation analyses focused on $\mathrm{NO}_{2}$, one of the most diffused and monitored air pollutant that is generally produced as a result of road traffic and other fossil fuel combustion processes. Data from the periods January - May 2019 and January - May 2020 were analyzed. The periods include the COVID-19 pandemic lockdown in Italy. This choice allowed a parallel exploration of the lockdown effects on air quality and provided insights into correlations between ground and satellite observations also under an anomalous emissions scenario. A significant reduction of $\mathrm{NO}_{2}$ concentrations was observed during the lockdown with respect to the same period of the previous year. Early results show a marked positive correlation on average between satellite and ground observations justifying the interest in further researching on the topic.

The paper continues as follows. Section 2 discusses assets and liabilities of air quality analyses from space by introducing state-of-art methods for employing satellite observations into ground-level air pollution monitoring. Section 3 describes data, correlation experiments and results for the Lombardy region case studies. Conclusion and future work are outlined in Section 4

\section{AIR QUALITY MONITORING FROM SPACE}

Concerning the technological aspects of air quality monitoring, significant advances have been achieved in recent years through the amelioration of ground sensors hardware performance and sampling accuracy, with a concurrent reduction of costs and sizes, of both fixed and portable equipment. However, the technological frontier that currently is most attracting the attention of both scientists and policymakers worldwide is the satellite remote sensing (Heal et al., 2012, Chance et al., 2019). Nowadays, atmospheric observations from space can provide daily high-resolution air pollutants estimates on a global scale. Satellite-based estimates are of utmost importance especially for rural areas with limited ground-level measurements, where they best promise to fill the data gap for assessing long-term human exposure, avoiding high economic investments by the managing authorities to increase the sensors networks density. Furthermore, satellite-based estimates can provide useful information on air pollution spatio-temporal dynamics even in areas where sensors density is high (Kim et al., 2020).

Satellite remote sensing for air quality monitoring has a long history starting from the ' 80 s with application to the analysis of large-scale air pollution episodes (Martin, 2008). This evolved in the current satellite constellations with higher spatial and temporal resolution capabilities (e.g. $5.5 \mathrm{~km}$ pixel resolution and 1 day revisit time provided by the Sentinel-5P) and the upcoming geostationary missions promising to further reduce the gap with the ground-based monitoring (Kim et al., 2020). As a consequence, air quality metrics and modelling are evolving to merge this increased data availability by coupling traditional models with data science techniques including machine-learning (Chen et al., 2019, Analitis et al., 2020, Liu et al., 2020). The fact is that satellite estimates are not direct measures of air pollutants but are derived from the spectral signatures of each atmospheric constituent by making numerical models a necessary mean to infer physical quantities out of them. The complex dynamics of air pollutants in the atmosphere further introduces uncertainties into models and - in turn - affects the reliability of satellite estimates especially to localscale air quality applications (Fowlie et al., 2019). In parallel to the improvement of satellite platforms and retrieval algorithms to infer physical quantities, the development of statistical methods to empower ground-level air quality monitoring with satellite estimates represents a key task to fully unlock the potential of atmospheric remote sensing into practical applications.

In general terms, statistical methods must be capable of modelling relationships between the ground measurements and satellite observations by providing a reliable estimation of air pollutants concentrations at locations without ground sensors (Liu et al., 2020). These relationships are highly variable both in space and time due to the concurrent influence of heterogeneous factors including land use characteristics, microclimatic conditions, and anthropogenic emissions patterns, among others. To that end, latest studies in the literature have proposed the adoption of machine-learning and geostatistics algorithms to account for complex correlations between air pollutants groundlevel concentrations, satellites estimates and possible informative covariates (Liu et al., 2020). Combined methods based on satellite estimates, including geographically and temporally weighted neural networks and random forests-based spatial regressions, have produced a general improvement in the prediction accuracy of ground-level air pollution (Chen et al., 2019, Li et al., 2020, Liu et al., 2020). The development of sophisticated and high-resolution models is favoured by the growing availability of air quality records (both from ground and satellites sensors) and ancillary covariates as open-data, as well as open software tools and infrastructure enabling outstanding opportunities for data retrieval and processing (Harris, Baumann, 2015).

Nevertheless, preliminary knowledge of complex air pollution spatio-temporal patterns remains critical to the development of the above methods. With this in mind, this works aims at investigating local space and time correlations between ground-level air pollutants measures and the Sentinel-5P satellite estimates (see Section 3). The final goal is to quantify underlying patterns in the data which may later be employed to select best modelling strategies to empower ground-level air quality monitoring with satellite observations.

\section{SENTINEL5-P AND GROUND-BASED OBSERVATIONS COMPARISON}

The case study selected for this work focuses on the correlation assessment between $\mathrm{NO}_{2}$ satellite estimates from the Sentinel5P and the authoritative ground measurements available for the Lombardy region (Northern Italy, $\sim 23.000 \mathrm{Km}^{2}$, see Figure 1a). The Lombardy region is characterized by an unfavourable geographical context for air pollution and ranks among the most polluted areas in Europe. Most of the population $(\sim 10$ million people) is located in the Po River basin (plain area) which is bordered on three sides by mountains that limit air 
mass exchanges (see Figure 1b). In addition, the Lombardy region counts many industrial facilities and intensive road transport activities strongly contributing to air pollution in the area (Carugno et al., 2016). $\mathrm{NO}_{2}$ emissions are primarily due to fossil fuel burning and, accordingly, the $\mathrm{NO}_{2}$ is among the most diffused air pollutants in the study region. Two analysis period were selected for the correlation analyses; respectively the months January - May in the years 2019 and 2020. Further than the computation of correlation between satellite and ground observations, the selected periods allowed for parallel quantification of COVID-19 lockdown effects on $\mathrm{NO}_{2}$, which from March 9 to May 18, 2020, caused a massive reduction of both industrial and transport activities in the Lombardy region.

\subsection{Data}

3.1.1 Gound-sensors data Air quality monitoring is performed in the Lombardy region by the Regional Environmental Protection Agency (ARPA Lombardia, https://www.arpalombardia.it) through its air quality sensors network. The network is composed of more than 100 fixed ground stations (see Figure 1a) providing hourly ground-level measures of pollutants concentrations $\left[\mu \mathrm{g} / \mathrm{m}^{3}\right]$, including $\mathrm{NO}_{2}$, $\mathrm{PM}_{10}, \mathrm{PM}_{2.5}, \mathrm{O}_{3}$, and $\mathrm{SO}_{2}$. Data are available starting from the year 2000 and they are freely distributed under CC-BY license through the Lombardy Region Open Data portal (https://www.dati.lombardia.it). Data can be downloaded in CSV format using the Open Data portal web interface or in JSON format through a dedicated open Application Programming Interface (API, http://dev.socrata.com).

$\mathrm{NO}_{2}$ hourly concentrations for the analysis periods the and sensors metadata (including geographic coordinates) were downloaded using the open API through Python scripting. 83 sensors were considered according to the availability of complete time-series measurements provided in the analysis periods. Data were arranged in tabular format (Pandas DataFrame) using the premier free and open Python library Pandas (https://pandas.pydata.org), and its spatial extension GeoPandas (https://geopandas.org). A single DataFrame containing $\mathrm{NO}_{2}$ hourly concentrations for each sensor was thus obtained and linked to a second DataFrame including sensors metadata.

3.1.2 Sentinel-5P data Satellite estimates of $\mathrm{NO}_{2}$ concentrations were retrieved from the European Space Agency (ESA) Sentinel-5P multispectral mission. The Sentinel-5P was launched on October 13, 2017, and represents the first mission of the European Copernicus Programme that delivers measurements of atmospheric constituents with the aim of improving the European Union's capacity for air quality monitoring (Koch, Bydekerke, 2018). Sentinel-5P data products are freely distributed by the Copernicus Programme and can be downloaded through its Data Access Hub (https://scihub.copernicus.eu). Data are available from mid-2018 and consists of Level-1B and Level-2 products providing a spatial resolution of $5.5 \mathrm{~km}$ (along-track) x $3.5 \mathrm{~km}$ (across-track) with global coverage and revisit time of 1 day. Level-2 products consist of troposphere trace gases estimates, including $\mathrm{NO}_{2}$, $\mathrm{O}_{3}, \mathrm{SO}_{2}$, carbon monoxide $(\mathrm{CO})$, methane $\left(\mathrm{CH}_{4}\right)$, and formaldehyde $\left(\mathrm{CH}_{2} \mathrm{O}\right)$, together with aerosol and could indexes. Data are distributed in NetCDF-4 format with an open-data license. A single full-orbit (2600 $\mathrm{Km}$ strip) Level-2 observation has a size in memory varying between 0.1 and 2 GB (depending on the product) and contains a variety of metadata such as quality bands and sensor parameters. The data products are provided

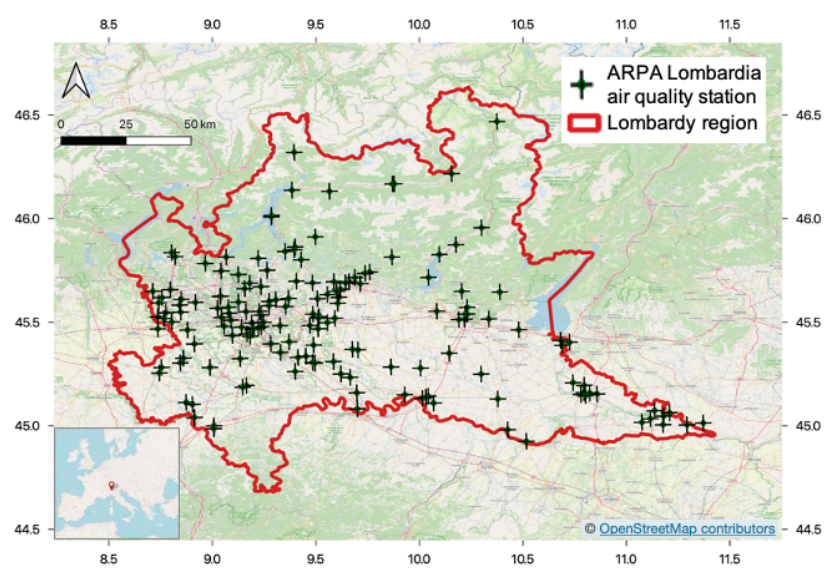

(a)

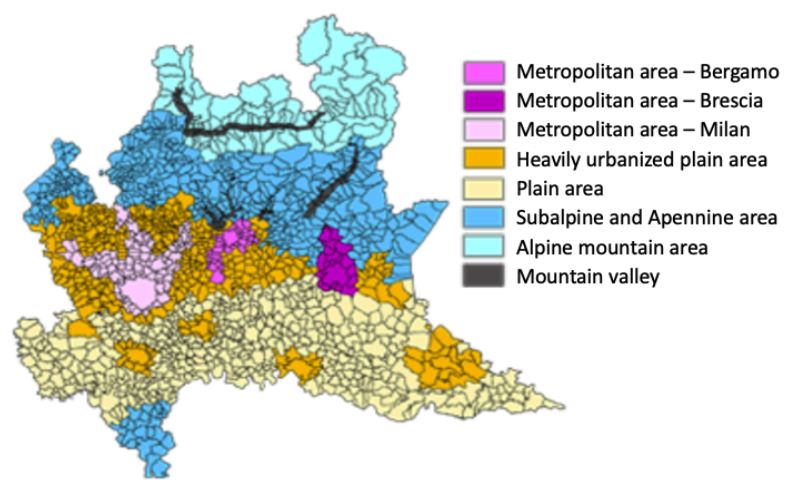

(b)

Figure 1. (a) Lombardy region area and ARPA Lombardia air quality sensors locations (coordinates reference system:

WGS84). (b) Lombardy region zonation schema adopted by ARPA Lombardia for air quality analysis (source:

https://tinyurl.com/yy538ruu). Metropolitan areas comprise the most populated cities in the region (Milan, Bergamo, and

Brescia)

in three different data streams: the near-real-time stream, the offline stream and the reprocessing stream. Near-real-time data are available within 3 hours after data acquisition and are meant for rapid use but provide lower quality as compared to the other data streams. Offline data are available within a few days after the acquisition as for the data final version from the reprocessed stream (Romahn et al., 2020). For long-term trend analysis, the offline and reprocessed data streams are suggested. Accordingly, the data used in the presented work was the Sentinel-5P Level-2 $\mathrm{NO}_{2}$ tropospheric column $\left[\mathrm{mol} / \mathrm{m}^{2}\right]$ product form the offline stream.

The NetCDF-4 files containing daily estimates of the $\mathrm{NO}_{2}$ tropospheric column were downloaded from the Copernicus Data Access Hub for the analysis periods. First, raw data were pre-processed to remove low-quality observations by considering only observations with a quality assurance value greater than 0.75 ( $q$ a value, available as metadata for each Sentinel-5P product). This quality threshold is suggested by (Romahn et al., 2020) to discriminate cloud-free and snow-ice free observations. Second, a geographic subset covering the Lombardy region was created for each of the Sentinel-5P product. Quality filters and the geographic subsets extraction were performed using the xArray Python library (http://xarray.pydata.org) along with the free and open-source software HARP - data har- 
monization toolset for scientific Earth observation data (https://github.com/stcorp/harp). Finally, time-series of $\mathrm{NO}_{2}$ tropospheric column daily observations were sampled from the pre-processed NetCDF files at each ARPA Lombardia sensor location and stored in a Pandas DataFrame.

\subsection{Data Processing}

The pre-processing operations produced two DataFrames containing geolocated time-series of $\mathrm{NO}_{2}$ concentrations $\left[\mu \mathrm{g} / \mathrm{m}^{3}\right.$ ] from ARPA Lombardia sensors (hourly observations) and $\mathrm{NO}_{2}$ tropospheric columns product of Sentinel-5P [mol $\left./ \mathrm{m}^{2}\right]$ (daily observations).

Daily averages were computed for the ARPA Lombardia sensors and compared between the two study periods to assess the COVID-19 lockdown effects on $\mathrm{NO}_{2}$ concentrations. Results are discussed in Section 3.3.1.

ARPA Lombardia sensors have an hourly sensing time period while the satellite passage each day happens between 10:00 AM to 1:00 PM GMT+0 according to the Sentinel-5P sunsynchronous orbit. An iterative procedure was implemented to average the hourly sensors' observations over a time-window and use this average for computing correlations with the satellite observations. The Pearson's correlation coefficient $\rho_{p}$ was computed from the pairwise comparison of each sensors timeseries and the corresponding satellite time-series, by averaging the hourly sensor observations on moving time-windows with different sizes (from 1 to 24 hours). $\rho_{p}$ ranges between 1 and -1 ; where 1 indicates a perfect positive correlation, 0 no correlation, and -1 perfect negative correlation (Schober et al., 2018). The average of $\rho_{p}$ coefficients obtained at each iteration (i.e. for each considered time-window position and size) was computed. The time-window that produced the strongest average correlation was identified by looking at the highest among the computed $\rho_{p}$ averages. For the presented case study, the selected time-window for averaging hourly observations of ARPA Lombardia sensor was between 10:00 AM to 2:00 PM GMT+0 which unsurprisingly overlaps the Sentinel-5P passage time range. Once the averaging time-window has been defined, $\rho_{p}$ was computed from each pair of time-series for all the sensors. Results are discussed in Section 3.3.2. It is worth noting that $\rho_{p}$ measures linear correlation which may represent a relevant limitation in assessing correlation between direct $\mathrm{NO}_{2}$ measures from ground sensors and satellite estimates derived from spectral signature analysis of the atmospheric column (Li et al., 2020).

To that end, an additional correlation coefficient was considered for assessing correlations disregarding the linear hypothesis. The Spearman's rank correlation coefficient $\rho_{s}$ was computed from each pair of time-series for all the sensors by considering the same time-windows adopted in the previous experiment. $\rho_{s}$ measures monotonic statistical dependence between ranks of two variables, thus allowing assessing correlations whether linear or not (Schober et al., 2018). $\rho_{s}$ ranges between 1 and -1 and values interpretation follows exacting the one of $\rho_{p}$. The Results from this second experiment are presented and compared to the previous ones in Section 3.3.2. Computations of both coefficients were performed by taking advantages of statistical functions included in the Python Pandas library.

\subsection{Results Discussion}

3.3.1 COVID-19 lockdown effect on $\mathrm{NO}_{2}$ A reduction of about $17.5 \%$ was observed through the comparison of daily av- erage $\mathrm{NO}_{2}$ concentrations between the COVID-19 lockdown period (March 9 - May 18, 2020) and the same period of the previous year (Figure 2). By considering only March, when the most restricted lockdown measures were ongoing, the reduction was of about $24.5 \%$. The results are aligned to the ones of previous studies carried out on the same topic for the Lombardy region (Collivignarelli et al., 2020). The sharp decrease of $\mathrm{NO}_{2}$ emissions reflects the massive reduction of industrial and transport activities imposed by the COVID-19 pandemic that affected the region.

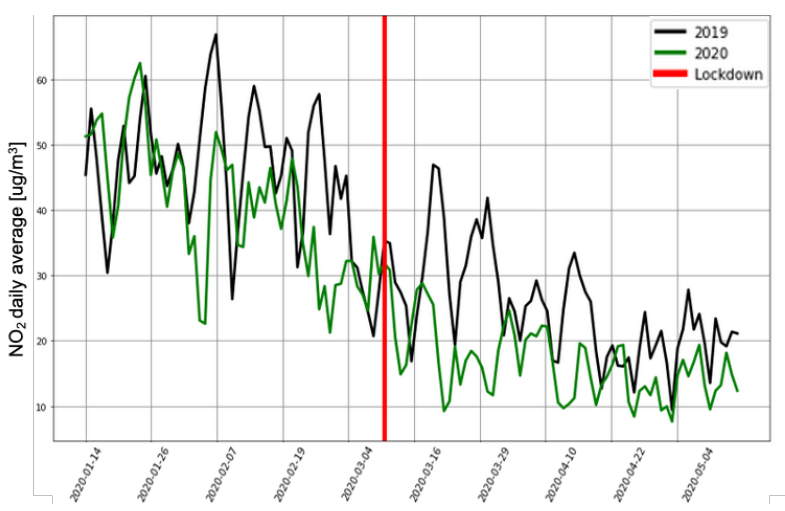

Figure 2. Comparison of the $\mathrm{NO}_{2}$ daily averages computed from the ARPA Lombardia sensors in the study periods. A substantial decrease in concentrations can be noticed during the

COVID-19 lockdown with respect to the previous year

3.3.2 Correlation analysis Results from the correlation analysis, carried out by means of $\rho_{p}$ and $\rho_{s}$ computations, are reported in Figure 3. Summary statistics of the two coefficients are included in Table 1. Results pointed out a global strong positive correlation between the $\mathrm{NO}_{2}$ ground measurements and the satellite estimates with mean $\rho_{p}$ and $\rho_{s}$ values near or larger than 0.75 . Correlations are stronger on average for the 2020 study period than 2019 but characterized by a higher standard deviation. Therefore, results suggest better agreement of satellite estimates with ground-level $\mathrm{NO}_{2}$ measurements at a lower concentration level (see Figure 2) or eventually at a lower emission level from the industry and transport sectors. Nevertheless, the computed standard deviations indicate a higher variability of the correlation coefficients among the different sensors in this anomalous emissions scenario.

Spatial patterns of correlation coefficients can be inferred from figures $3 \mathrm{a}, 3 \mathrm{~b}, 3 \mathrm{c}$, and $3 \mathrm{~d}$. It can be noticed that the lowest correlations are mostly confined in the mountain areas and valleys while the highest correlations are mostly found in the plain and metropolitan areas (refer to Figure $1 \mathrm{~b}$ for the zonation schema). This might be connected to one or a combination of different factors. First, the orography of the mountain areas and valleys might obstacles the satellite view on the lower atmosphere column by biasing the satellite estimates with respect to ground measures. Second, microclimatic conditions, background emissions, emission sources, and general landscape characteristics (e.g. the land use) of mountain areas are generally different than the plain area and this might influence the $\mathrm{NO}_{2}$ ground concentrations (Chen et al., 2019). Investigations on the above matter are out of scope for the presented case study and require further analysis to be confirmed.

From the summary statistics of $\rho_{p}$ and $\rho_{s}$ (Table 1), a final relevant insight was obtained. Namely, $\rho_{s}$ outperformed $\rho_{p}$ in 
terms of mean and median, thus suggesting non-linear prediction models being more adequate to infer $\mathrm{NO}_{2}$ ground concentrations using the Sentinel-5P satellite estimates. This insight is critical for future activities of the presented work, which aim at developing satellite-based local air quality prediction models.

\begin{tabular}{|c|c|c|c|c|}
\cline { 2 - 5 } \multicolumn{1}{c|}{} & \multicolumn{2}{c|}{$\rho_{p}$} & \multicolumn{2}{c|}{$\rho_{s}$} \\
\cline { 2 - 5 } \multicolumn{1}{c|}{} & $\mathbf{2 0 1 9}$ & $\mathbf{2 0 2 0}$ & $\mathbf{2 0 1 9}$ & $\mathbf{2 0 2 0}$ \\
\hline mean & 0.726 & 0.763 & 0.747 & 0.795 \\
\hline median & 0.753 & 0.795 & 0.787 & 0.837 \\
\hline standard deviation & 0.118 & 0.151 & 0.121 & 0.141 \\
\hline
\end{tabular}

Table 1. Summary statistics for Pearson's correlation coefficients $\left(\rho_{p}\right)$ and Spearman's rank correlation coefficients

$\left(\rho_{s}\right)$ between $\mathrm{NO}_{2}$ concentration time-series of ARPA Lombardia sensors and the Sentinel-5P

\section{CONCLUSIONS AND FUTURE WORK}

In the presented study, an analysis of correlations between ground sensors measurements (ARPA Lombardia) and satellite estimates (Sentinel-5P) time-series of $\mathrm{NO}_{2}$ air concentrations in the Lombardy region was carried out. The analysis considered two equivalent study periods in the months JanuaryMay of years 2019 and 2020. The study periods included the COVID-19 lockdown in Italy. The analysis settings allowed to investigate local spatio-temporal correlation patterns between ground sensors measurements and satellite estimates by also exploring the lockdown effects on $\mathrm{NO}_{2}$ emissions and providing insights into correlations under an anomalous emissions scenario.

Results indicate a reduction of about $17.5 \%$ for the daily average $\mathrm{NO}_{2}$ concentrations in the Lombardy region during the lockdown period with respect to the same period of the previous year. The analysis points out a strong positive correlation between ground sensors measurements and satellite estimates with mean $\rho_{p}$ and $\rho_{s}$ values near or larger than $0.75 . \rho_{s}$ values outperform on average $\rho_{p}$ by suggesting not-linear correlation being more effective than linear one for inferring $\mathrm{NO}_{2}$ ground concentrations using Sentinel-5P observations. Correlations are generally stronger in plain and metropolitan areas than in the mountain areas thus remarking the need for considering local landscape and microclimatic conditions in the development of satellite-based air quality predictive models (Chen et al., 2019). This preliminary work focused on $\mathrm{NO}_{2}$ while it can be directly extended to other common air pollutants such as $\mathrm{O}_{3}$ and $\mathrm{SO}_{2}$.

The presented exploratory analysis is a propaedeutic step for future work which will focus on the development of prediction models to empower the regional air quality monitoring with satellite observations assets. The models will take the best advantage of machine-learning and geostatistics methods to allow accounting for both non-linear correlations between satellite estimates and ground-measures as well as local spatio-temporal covariates (e.g. microclimatic conditions, land use, etc.) affecting air pollutant concentrations. The ultimate goal is to improve the granularity of the available ground-based air quality measurements for air quality monitoring and, at the same time, enabling exposure models to better account for time and space variability of exposure patterns. The extensive adoption of open data, as well as the exclusive use of free and open-source software, provide the analysis with a potential to be empowered, replicated, and improved; thus favouring the scientific debate and benchmarking on the addressed topics.

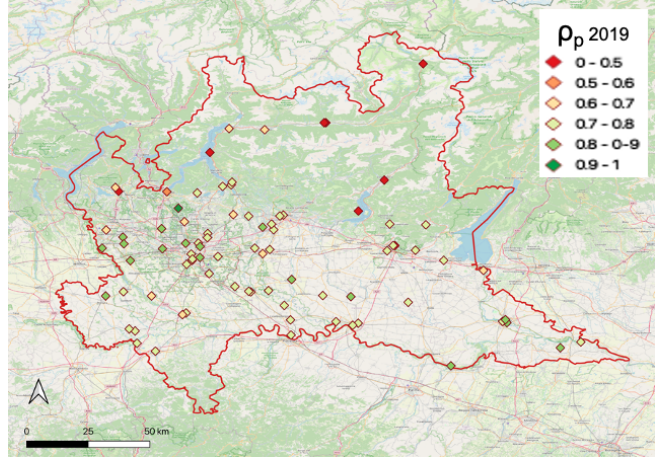

(a)

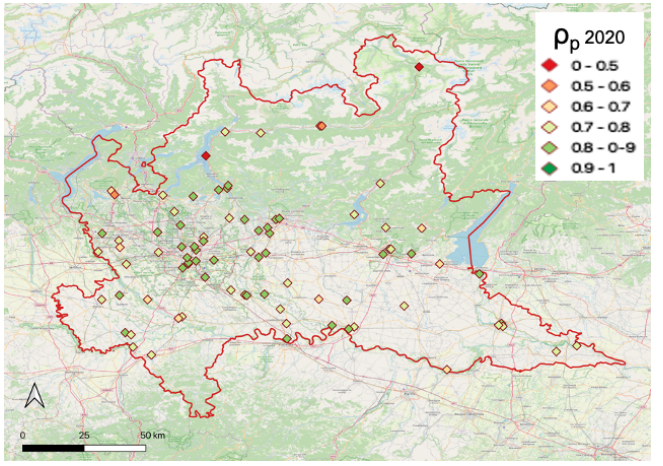

(b)

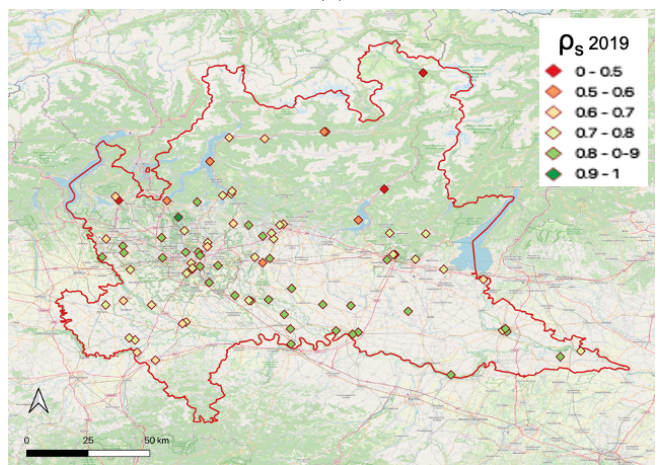

(c)

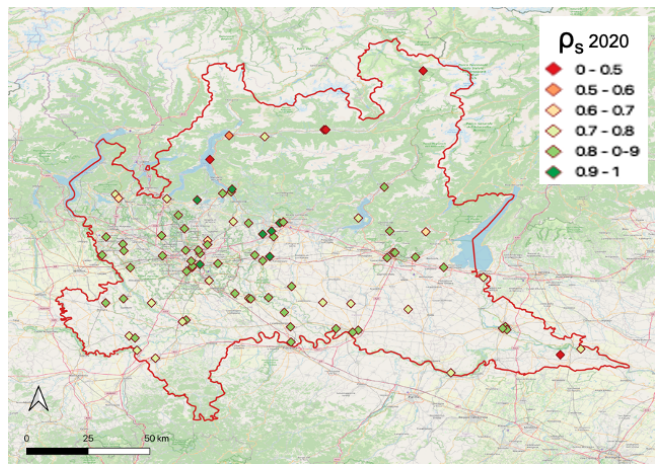

(d)

Figure 3. Maps of Pearson's correlation coefficients $\left(\rho_{p}\right)$ at each sensor location, computed from the comparison of $\mathrm{NO}_{2}$ concentration time-series of ARPA Lombardia sensors and the Sentinel-5P in the two analysis periods, 2019 (a) and 2020 (b). Maps of Spearman's rank correlation coefficients $\left(\rho_{s}\right)$ computed on the same data in the two analysis periods, 2019 (c) and 2020 
There are pending questions about the accuracy and precision of satellite-based estimates (not direct measures) and their actual role in the operational air quality monitoring, especially within the current air quality regulatory framework (Fowlie et al., 2019). Indeed, daily observations provided by the satellites are still not suitable for computing most of air quality indicators required by both national and international legislation (WHO, 2006). The improvement of satellite platforms sampling frequency and accuracy as well as of air quality modelling techniques is key to tackle these issues. In view of the above, the outlined work aims at contributing to the baseline research on ground and satellite sensors interplay for air quality monitoring that is advised by the international scientific community to reduce accuracy and reliability gaps and contribute to nextgeneration air quality satellite missions.

\section{REFERENCES}

Analitis, A., Barratt, B., Green, D., Beddows, A., Samoli, E., Schwartz, J., Katsouyanni, K., 2020. Prediction of PM2. 5 concentrations at the locations of monitoring sites measuring PM10 and NOx, using generalized additive models and machine learning methods: A case study in London. Atmospheric Environment, 240, 117757.

Brunekreef, B., Holgate, S. T., 2002. Air pollution and health. The lancet, 360(9341), 1233-1242.

Carugno, M., Consonni, D., Randi, G., Catelan, D., Grisotto, L., Bertazzi, P. A., Biggeri, A., Baccini, M., 2016. Air pollution exposure, cause-specific deaths and hospitalizations in a highly polluted Italian region. Environmental research, 147, 415-424.

Chance, K., Liu, X., Miller, C. C., Abad, G. G., Huang, G., Nowlan, C., Souri, A., Suleiman, R., Sun, K., Wang, H. et al., 2019. Tempo green paper: Chemistry, physics, and meteorology experiments with the tropospheric emissions: monitoring of pollution instrument. Sensors, Systems, and Next-Generation Satellites XXIII, 11151, International Society for Optics and Photonics, 111510B.

Chen, Z.-Y., Zhang, R., Zhang, T.-H., Ou, C.-Q., Guo, Y., 2019. A kriging-calibrated machine learning method for estimating daily ground-level NO2 in mainland China. Science of The Total Environment, 690, 556-564.

Collivignarelli, M. C., Abbà, A., Bertanza, G., Pedrazzani, R., Ricciardi, P., Miino, M. C., 2020. Lockdown for CoViD-2019 in Milan: What are the effects on air quality? Science of The Total Environment, 732, 139280.

Duque, L., Relvas, H., Silveira, C., Ferreira, J., Monteiro, A., Gama, C., Rafael, S., Freitas, S., Borrego, C., Miranda, A., 2016. Evaluating strategies to reduce urban air pollution. Atmospheric Environment, 127, 196-204.

Fowlie, M., Rubin, E., Walker, R., 2019. Bringing satellitebased air quality estimates down to earth. AEA Papers and Proceedings, 109, 283-88.

Harris, R., Baumann, I., 2015. Open data policies and satellite Earth observation. Space Policy, 32, 44-53.

Heal, M. R., Kumar, P., Harrison, R. M., 2012. Particles, air quality, policy and health. Chemical Society Reviews, 41(19), 6606-6630.
Kim, J., Jeong, U., Ahn, M.-H., Kim, J. H., Park, R. J., Lee, H., Song, C. H., Choi, Y.-S., Lee, K.-H., Yoo, J.-M. et al., 2020. New era of air quality monitoring from space: Geostationary Environment Monitoring Spectrometer (GEMS). Bulletin of the American Meteorological Society, 101(1), E1-E22.

Koch, A. C., Bydekerke, L., 2018. Sentinel 5p in the framework of the EU Copernicus programme. EGUGA, 6557.

Kuklinska, K., Wolska, L., Namiesnik, J., 2015. Air quality policy in the US and the EU-a review. Atmospheric Pollution Research, 6(1), 129-137.

Li, T., Shen, H., Yuan, Q., Zhang, L., 2020. Geographically and temporally weighted neural networks for satellite-based mapping of ground-level PM2. 5. ISPRS Journal of Photogrammetry and Remote Sensing, 167, 178-188.

Liu, Y., Cao, G., Zhao, N., 2020. Integrate machine learning and geostatistics for high-resolution mapping of ground-level pm2. 5 concentrations. Spatiotemporal Analysis of Air Pollution and Its Application in Public Health, Elsevier, 135-151.

Martin, R. V., 2008. Satellite remote sensing of surface air quality. Atmospheric environment, 42(34), 7823-7843.

Rafaj, P., Kiesewetter, G., Gül, T., Schöpp, W., Cofala, J., Klimont, Z., Purohit, P., Heyes, C., Amann, M., Borken-Kleefeld, J. et al., 2018. Outlook for clean air in the context of sustainable development goals. Global Environmental Change, 53, 1-11.

Romahn, F., Pedergnana, M., Loyola, D., Apituley, A., Sneep, M., Veefkind, J. P., Argyrouli, A., Lutz, R., 2020. Sentinel-5 precursor/tropomilevel 2 product user manualcloud properties. Technical report, available at: https://sentinel.esa.int/documents/247904/2474726/Sentinel5P-Level-2-Product-User-Manual-Cloud.

Schober, P., Boer, C., Schwarte, L. A., 2018. Correlation coefficients: appropriate use and interpretation. Anesthesia \& Analgesia, 126(5), 1763-1768.

WHO, 2006. Who air quality guidelines for particulate matter, ozone, nitrogen dioxide and sulfur dioxide: global update 2005: summary of risk assessment. Technical report, World Health Organization. 